\title{
EDITORIAL
}

\section{Image and sound}

Organised Sound has until now been principally concerned with the audio arts. However, we live in a rapidly changing world; in particular, given the relentless evolution of competent technology in the form of multimedia PCs, new rendering technologies and standardised distributive media such as the web and MPEG, it seems timely to explore the relationship between the audio and visual spheres in the digital arts.

Not to do so risks de facto acceptance of misguided dogma-the supposed predominance of the one medium over the other, for instance. Whilst people have wrestled with these issues in the past, the imminent arrival of this technology on a large scale into consumer markets creates new opportunities, as well as an urgency to deal with aesthetic concerns: what are the compositional issues, given that we may be dealing with audiovisual artefacts; what is the meaning of performance of such artefacts; what tools exist or need to be developed for such composition and performance; what is our experience of good (and bad) practice in this field; what is our attitude to a new consumer multimedia 'product' which could be distributed on an unprecedented scale over the Internet? These are examples of the aspects which we set out to explore in this issue of Organised Sound, especially in relation to our established sphere of interest concerning electroacoustic music.

We are at the beginning of a new era in the digital arts and there is a sense that the authors presenting papers in this issue are pioneers in the field. Laurie Spiegel's paper 'Graphical GROOVE' sets us off with a historical perspective, describing her pioneering work producing coherent 'sound and image' compositions in the 1970s. Her work progressed against considerable technological difficulties caused by the primitive integration of image- and sound-rendering systems available at that time. We share her sense of frustration when further development of her work was made inaccessible by the problem of obsolescent hardware.

It is interesting to compare her experiences of Graphical GROOVE with the work described in the paper by Andy Hunt and his colleagues. In many ways, this work can be regarded as a continuation of the approach adopted by Laurie Spiegel, but with the important emphasis on integration of image and sound rendering made possible by the MIDAS system. Although the authors would not claim to expound a developed aesthetic for audiovisual art, their provision of interactive exploration of image within a model of composition and performance may well lead the way to the evolution of one such aesthetic. Their use of open distributed processing should also help with the problem of obsolescence experienced by Laurie.

It is important in an issue such as this to receive reports describing successful works of digital art. We therefore welcome the contribution of several authors describing the implementation of their compositions and installations.

Jøran Rudi presents the processes employed in the realisation of his audiovisual pieces Concrete Net, Planet and When Timbre Comes Apart. His approach to detailed rendering of image over prolonged periods of time using considerable computing resources provides a contrast to the interactive approach used by other contributors to the issue.

The theme of interaction is taken up in different ways by authors of other papers in this issue. Natasha Barrett and Oyvind Hammer in their Mimetic Dynamics installation, allow both artist and viewer to explore the relationship between real (physical) processes and mathematical models of the processes. The viewer is invited to interact with the installation, and thus modify its subsequent evolution. Tapio Lokki and his colleagues describe their DIVA system which uses gestural recognition processing to allow the viewer to interact with a virtual reality model of an orchestra in a virtual concert hall. This has immediate application in the architectural design of acoustic spaces, but perhaps also points the way to the use of virtual reality as a means to provide interactive exploration of audiovisual art.

Finally, not forgetting our roots in electroacoustic music, we welcome an article by Eduardo Reck Miranda describing the realisation of his piece Requiem per una veu perduda-a piece for mezzo-soprano accompanied by effects processing and other recorded electroacoustic material.

We publish examples of images produced by the works and systems described in this issue. Constrained, as we are, by the static greyscale image of 
the monochrome printed page, it is difficult to do justice to the beauty of the images in their use of colour, and especially dynamics made available by the new technologies. We therefore encourage readers to investigate the various Web sites referenced in the papers to experience something of the true essence of the compositions described, and especially to interact with the evolution of this new and exciting art. 